\title{
Review: palliative chemotherapy reduces death and progression at 12 months in advanced or metastatic colorectal cancer
}

Colorectal Meta-analysis Collaboration. Palliative chemotherapy for advanced or metastatic colo-rectal cancer. Cochrane
Database Syst Rev 2000;(2):CD001545 (latest version 16 Nov 1999).

\section{QUESTION: In patients with locally advanced or meta-static colorectal cancer, what are the benefits and harms of palliative chemotherapy?}

\section{Data sources}

Studies were identified by searching Medline, EMBASE/ Excerpta Medica, CancerLit, the Cochrane Controlled Trials Register, CINAHL, HealthSTAR, Science Citation Index, Edina Biosis, NHS Economic Evaluation Database, Index to Scientific and Technical Proceedings, and PASCAL (to October 1998); handsearching conference abstracts; scanning bibliographies of relevant studies; searching sources of unpublished trials; and contacting authors.

\section{Study selection}

Studies in any language were selected if they were randomised controlled trials (RCTs) that compared palliative chemotherapy with supportive care alone in patients with advanced or metastatic colorectal cancer.

\section{Data extraction}

The quality of studies was assessed by using the Jadad scale. Authors were contacted for individual patient data (baseline patient characteristics, allocated treatment group, date of randomisation, tumour response, survival and progression status, and date of death or last follow up). 2 reviewers extracted data from published studies, and meta-analysis was done by using both published data and individual patient data.

\section{Main results}

13 RCTs (1365 patients) met the selection criteria. Individual patient data were obtained for 7 RCTs $(866$ patients). Meta-analysis of published data showed that palliative chemotherapy (5-fluorouracil [5-FU], alone or in combination; fluoridine; irinotecan; or tauromustine) led to a reduction in death and progression at 12 months (table). Statistically significant heterogeneity existed among these RCTs. Studies with individual patient data were not heterogeneous; they also showed a reduction in death (7 RCTs; number needed to treat [NNT] $6,95 \%$ CI 4 to 11 ) and progression (3 RCTs, NNT 4) at 12 months. The evidence for toxicity, quality of life, and symptom control was inconclusive because of inconsistent reporting and use of poor quality methods.

\section{Conclusions}

In patients with locally advanced or metastatic colorectal cancer, palliative chemotherapy reduces death and progression at 12 months. The evidence on toxicity, symptom control, and quality of life is inconclusive.

\section{COMMENTARY} 6 mo in median progression free survival). allowed chemotherapy at the onset. chemotherapy in colorectal cancer. 1998;16:301-8. Meet Am Soc Clin Oncol 1999;18:A1010. Soc Clin Oncol 2000;18:A938.
Source of funding: NHS National Cancer Research E Development Programme UK.

For correspondence: Dr L Y Best, CRX Wessex Medical Oncology Unit, Southampton General Hospital, Level F (809), Centre Block, Southampton SO16 6YD, UK. Fax +44 (0)1703783839.

Palliative chemotherapy $v$ supportive care for advanced or metastatic colorectal cancer*

\begin{tabular}{|c|c|c|c|c|c|}
\hline \multirow[b]{2}{*}{$\begin{array}{l}\text { Outcomes } \\
\text { at } 12 \\
\text { months }\end{array}$} & \multirow[b]{2}{*}{$\begin{array}{l}\text { Number } \\
\text { of } \\
\text { studies }\end{array}$} & \multicolumn{2}{|c|}{ Weighted event rates } & \multirow[b]{2}{*}{ RRR $(95 \% \mathrm{Cl})$} & \multirow[b]{2}{*}{ NNT (CI) } \\
\hline & & Chemotherapy & $\begin{array}{l}\text { Supportive } \\
\text { care }\end{array}$ & & \\
\hline Death & 10 & $53 \%$ & $68 \%$ & $22 \%$ (7 to 34 ) & 7 (5 to 20 ) \\
\hline Progression & 4 & $66 \%$ & $76 \%$ & $14 \%$ (4 to 23$)$ & 10 (6 to 36$)$ \\
\hline
\end{tabular}

This well conducted meta-analysis by the Colorectal Meta-analysis Collaboration supports the view that palliative chemotherapy is beneficial in treating colorectal cancer. Progression free survival is prolonged, and deaths are reduced at 12 months. The benefits are substantial and clinically important. When studies with individual patient data are analysed, relative risk reductions of 35\% in deaths and $49 \%$ in progression at 12 months are shown. This finding translates into a $16 \%$ absolute difference in survival and a 25\% increase in progression free survival (increases of 3.7 mo in median survival and

This benefit is probably underestimated. An unknown proportion of patients randomly allocated to supportive care received delayed chemotherapy, and 3 trials

The issue is not whether to treat but when and how it is best to treat. Other potential modalities that either improve outcome or decrease toxicity include the use of continuous infusion 5-FU, oral 5-FU, ${ }^{2}$ and combination chemotherapy. ${ }^{3}$ Future studies that incorporate measures of quality of life will better define the role of palliative

Edmond Chouinard, MD Hamilton Regional Cancer Centre Hamilton, Ontario, Canada

1 Meta-analysis Group in Cancer. Efficacy of intravenous continuous infusion of fluorouracil compared with bolus administration in advanced colorectal cancer. J Clin Oncol

2 Twelves C, Harper P, Van Cutsem E, et al. A phase III trial (SO14796) of xeloda (capecitabine) in previously untreated advanced/metastatic colorectal cancer [abstract]. Proc Annu

3 Saltz LB, Douillard J, Pirotta N, et al. Combined analysis of two phase III randomized trials comparing irinotecan (C), fluorouracil (F), leucovorin (L) vs F alone as first-line therapy of previously untreated metastatic colorectal cancer (MCRC) [abstract]. Proc Annu Meet Am 